\title{
A Simulation Based Mobility Models Comparative Study
}

\author{
C. Joumaa \\ SeT Lab, UTBM \\ 90010 Belfort Cedex \\ France \\ +33 (0)3 84583823 \\ chibli.joumaa@utbm.fr
}

\author{
L. Moalic \\ SeT Lab, UTBM \\ 90010 Belfort Cedex \\ France \\ +33 (0)384583829 \\ laurent.moalic@utbm.fr
}

\author{
S.Lamrous \\ SeT Lab, UTBM \\ 90010 Belfort Cedex \\ France \\ +33 (0)3 84583253 \\ sid.lamrous@utbm.fr
}

\author{
A.Caminada \\ SeT Lab, UTBM \\ 90010 Belfort Cedex \\ France \\ +33 (0)3845834 46
}

alexandre.caminada@utbm.fr

\begin{abstract}
This paper presents a comparative study between several mobility models. Simulation environment is a time variant real representation of a city based on GIS, integrating all geographical and socio-economical information relative to this city. Simulation results are the basis of the comparative analysis between the models. This comparison is made by setting metrics evaluating individual and population displacement and that by quantifying each model's degree of realism.
\end{abstract}

\section{Keywords}

Mobility Models, Individual Motion, Population Flow, Comparison Metrics, Simulation.

\section{INTRODUCTION}

Mobility models are a key element in simulating human (individual/population) motion and displacement. These models became a necessity with the emergence of mobile networks in the last century, and all the mobile services proposed or to be proposed in the future. But with the large number of emerging mobility models; how can we choose the best adapted model for a given application?

The aim of the work presented in this paper is to perform a comparison between different well known mobility models divided into two major classes: random models and terrain aware models. Random Waypoint Mobility Model [1, 2], Random Walk Mobility Model [3], Normal Walk Mobility Model [4, 5], Smooth Mobility Model [6, 7], are the random models. And the terrain aware models are the Markovian Movement Model [8, 9], Normal Markovian Mobility Model and the Mask Based [10, 11, 12] Mobility Model.

Permission to make digital or hard copies of all or part of this work for personal or classroom use is granted without fee provided that copies are not made or distributed for profit or commercial advantage and that copies bear this notice and the full citation on the first page. To copy otherwise, or republish, to post on servers or to redistribute to lists, requires prior specific permission and/or a fee.

SIMUTOOLS'09, Mars 2-6, 2009, Rome, Italy.

Copyright 2009 ICST, ISBN 978-963-9799-45-5.

\section{SIMULATION ENVIRONMENT}

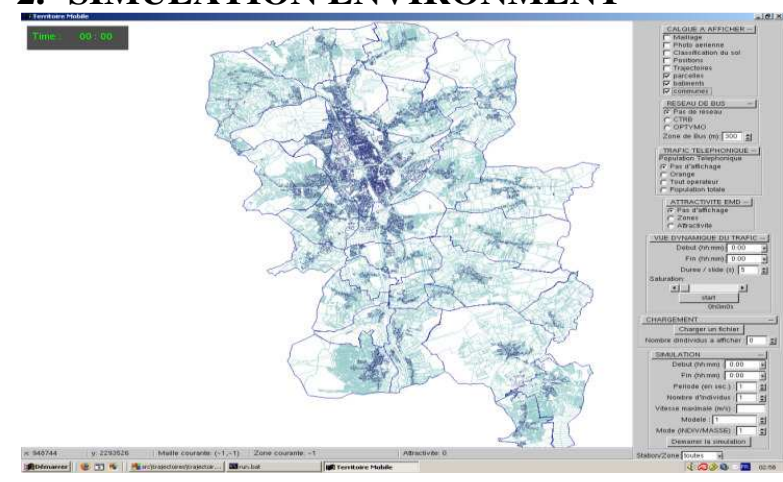

Figure 1. General view of the simulation platform

Individual trajectories and population motion are simulated in a real environment representing the city of Belfort in France. Several simulation runs are performed for each of the mobility models enumerated previously.

Based on these simulations an evaluation of each model is done by quantifying its characteristics and evaluating them in metrics.

This platform is a representation of Belfort, a city in the east of France, as shown in Figure 1.

Several layers of data were used to reproduce a real environment, such as GIS shapefiles, survey data and socio-economical information collected by professionals for regional planning needs.

A layer of data is the aggregation of all the provided data. This layer is necessary for the simulation of mobility models taking into account the ground characteristics. The environment is divided into square grid cells, with a side length equal to twenty five meters. Each of these grid cells is characterized by a value computed after the data classification based the dominant structure present in this grid cell and all other sources of information, this value is called the attraction weight. 


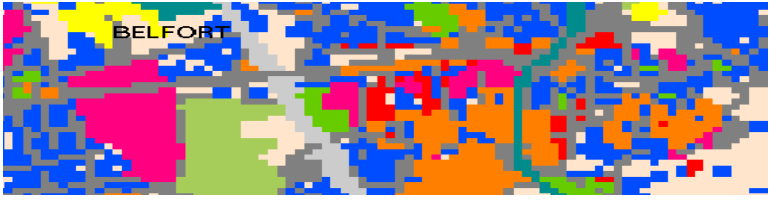

Figure 2. Ground Classification view mode of a part of the city of Belfort (each color corresponds to a class)

\section{SIMULATIONS AND RESULTS}

For trajectories one individual trajectory was simulated for each mobility model. The displacement speed was fixed. And the simulation time is set to eighteen hours from 6 am to $0 \mathrm{am}$.

As for population comparison, 75877 individuals corresponding to the real population of this region were simulated for each mobility model. Individuals were moving at constant speed. And simulation period was as for the trajectory case set to eighteen hours.

In order to compare all the previously mentioned mobility models we need to conduct two series of tests: individual and population simulation tests. A series of metrics was developed in order to establish a full comparison taking into account each and every aspect of all the implemented mobility models, for each of the two test types.

For trajectory comparison we decomposed a trajectory into two major parts: a curl (a concentration of several positions in a reduced space) and non-curl (path between two curls). A good trajectory is that that contains curls that are concentrated on activity structures identified by the ground classification, and as straight as possible non-curl paths connecting these curls.

A general metric was also set using all metrics to analyze the overall trajectory.

The aim of the population metrics is to evaluate each and every cloud or mass concentration generated with any of the previously presented mobility models.

The simulation time is divided to several periods. For each simulation period generated population convergence positions are compared with real traffic location.

Results presented that the final positions of the population simulation are better for terrain aware models, with better performance for positions generated using the Normal Markovian Mobility Model. This was clear in the population simulation figures, shown previously.

\section{CONCLUSION}

The metrics in this study were created to evaluate and compare mobility models independently from the possible applications. The aim was to evaluate all the mobility features that each of the models give.

The study began with the development of the simulation environment. Then after presenting the mobility models and their trajectory and population simulation, metrics were elaborated.

The results came as we expected, terrain aware models gave the best performance.

Future works for this study would be to compare simulated trajectories with real individual traces, and simulated population motion with real distribution measured data.

\section{REFERENCES}

[1] William Navidi, Tracy Camp, Stationnary Distributions for the Random Waypoint Mobility Model, - IEEE Transactions on Mobile Computing, 2004.

[2] Tracy Camp, Jeff Boleng, Vanessa Davies, A Survey of Mobility Models for Ad hoc Network Researchs, Wireless Communications F4 Mobile Computing (WCMC): Special issue on Mobile Ad Hoc Networking: Research, Trends and Applications, 2(5):483 502, 2002.

[3] Santashil PalChaudhuri, Jean-Yves Le Boudec, Milan Vojnovic, Perfect Simulation for Random Trip Mobility Models, Proceedings of IEEE Infocom 2005

[4] Chiu-Ching Tuan, Chen-Chan Yang, A Normal Walk Model for Mesh PCS Networks, Proceedings of the 18th International Conference on Advanced Information Networking and Appplications, Volume 2 AINA'04,IEEE Computer Society, March 2004

[5] Chiu-Ching Tuan, Chen-Chan Yang, A compact Norma Walk Model for PCS Networks, Proceedings of the 18th International Conference on Advanced Information Networking and Applications, Volume 2 AINA'04, IEE Computer Society, March 2004

[6] Christian Bettstetter, Mobility Modeling in Wireless Networks: Categorization, Smooth Movement, and Border Effects, ACM SIGMOBILE Computing and Communication Review, Volume 5, Issue 3, pp 55-56, ACM Press 2001

[7] Christian Bettstetter, Smooth is better than Sharp: A random Mobility Model for Simulation of Wireless Networks, Proceedins of the 4th ACM international workshop on Modeling, analysis and simulation of wireless and mobile systems, ACM press, 2001

[8] A. Burulitisz, S. Imre, and S. Szabo, On the accuracy of mobility modelling in wireless networks, presented at IEEE International Conference on Communications, 2004

[9] A. Bar-Noy, I. Kessler, and M. Sidi, Mobile users: to update or not to update? presented at INFOCOM '94. 13th Proceedings IEEE of Networking for Global Communications., Toronto, Ont, 1994

[10] C. Joumaa, A. Caminada, S. Lamrous, Mask Based Mobility Model A new mobility model with smooth trajectories, SpaSWiN 2007, Limassol, Cyprus, April, 2007.

[11] C. Joumaa, A. Caminada, S. Lamrous, Mask Based Mobility Model: A 2-D indoor 3-D outdoor mobility model, EMC Europe Workshop 2007, Paris, France, June 2007.

[12] C. Joumaa, A. Caminada, S. Lamrous, Mobility simulation for the evaluation of UMTS power control algorithms, NTMS 08, Tangier Morocco, November 2008 